\title{
Estimation of Some Biomarkers and Cholesterol / HDL Ratio to Predict the Risk of Cardiovascular Disease in Rheumatoid Arthritis patients
}

\author{
Enas Abdul Kareem Jabbar \\ enaskareemjj0@gmail.com \\ Department of Basic Medical \\ Science, College of Nursing, \\ University of Thi Qar, Iraq.
}

\author{
Shaymaa Z. Al-Rumaidh \\ shaymaaalrumaidh@gmail.com \\ College of Science,University of \\ Thi-Qar, Iraq.
}

\author{
Jamela Jouda \\ jamela.jouda@uomustansir \\ iyaha.idu.iq \\ Department of Biology, \\ College of Science, \\ Mustansiriyiah University, \\ Bagdad, Iraq.
}

\begin{abstract}
Rheumatoid arthritis (AR) is one of the chronic diseases resulting in many complications such as cardiovascular disease (CVD). Any change in the lipid profiles and myocardial markers indicates cardiovascular disease risk, so this study is designed to monitor the pattern of lipid profiles and myocardial markers in newly diagnosed RA patients. Blood samples were collected from 70 Iraqi patients newly diagnosed with rheumatoid arthritis (male and female) and 30 healthy served as control. These individuals were aged 35-65 years. The serum samples were obtained to determine myocardial markers; included troponin, creatinine kinase (CK), lactate dehydrogenase (LDH), and glutamic oxaloacetic transaminase GOT; and lipid profiles; such as cholesterol, triglyceride, high-density lipoprotein (HDL), low-density lipoprotein (LDL), and very-low-density lipoprotein (VLDL); using a kit from Roch (Germany), measured automatically with Minividas, Biomerieux (France). No differences between levels of all lipid profiles and myocardial markers in newly diagnosed RA patients compared with the healthy group were found as well as they were within the normal values, but interestingly, the cholesterol/HDL ratio increased significantly in RA patients comparing with healthy, so it could conclude that the risk of CVD could be increase also among the newly diagnosed of RA patients. Moreover, the cholesterol/HDL ratio should be probably included in a model to predict the risk of cardiovascular disease for RA in addition to the gender, age at the disease onset, and severity markers of disease.
\end{abstract}

Keywords: Cardiovascular disease risk, myocardial biomarkers, lipid profile, rheumatoid arthritis disease, TC/HDL ratio.

\section{Introduction}

One of the multiple systemic chronic diseases is rheumatoid arthritis (RA) which is usually characterized as heterogeneous symptoms, variable disease progression, and extracurricular manifestations [1]. One of the complications of this disease is the risk of developing cardiovascular disease (CVD), where studies indicate that the risk of accidental cardiovascular disease has increased by $48 \%$ in previously diagnosed patients with rheumatoid arthritis compared to the individuals without RA [2].

Clinical reports define many markers that could detect the transaminases released from dying myocytes, help diagnosis of myocardial infarction, prognosis, and risk stratification of For more information about the Conference please visit the websites: 
patients with the potential cardiovascular disease. These markers included troponin, creatinine kinase (CK), glutamic oxaloacetic transaminase GOT, and Lactate dehydrogenase (LDH). All these enzymes are released in different quantities by the death of muscle cells, but the research continued since the sensitivity and specificity for myocardial necrosis fuels are lacking [3].

Many studies have reported lipid abnormalities in previously diagnosed RA patients $[4,5$, 6] one of the traditional cardiovascular risk factors [7]. Dyslipidemia is observed in these patients differently than in the general population, including total cholesterol and HDL $[8,9]$. Since the ratio of disproportionate between TC and HDL levels increases the index of atherogenic, it is an important predictive sign of CVD [1].

These pieces of evidence proved that the previously diagnosed patients with RD could have lipid and myocardial markers abnormalities while to our knowledge there is no research study on these markers in newly diagnosed patients with $\mathrm{RD}$, so this study is designed to monitor the pattern of lipid profiles and myocardial markers in newly diagnosed RA patients.

\section{Materials Method}

Seventy newly diagnosed Iraqi patients with rheumatoid arthritis and thirty healthy (male and female) are recruited from the al-Husain hospital in Thi-Qar city at age of 35-65 years. Five ml blood was collected from the antecubital vein of each of the patients and healthy and put in dry sterilized test tubes. The blood samples were centrifuged for $5 \mathrm{~min}$ at 3000rpm to obtain the serum that used to determine myocardial markers; included troponin, CK, LDH, and GOT; and lipid profiles; included cholesterol, triglyceride, HDL, LDL, and VLDL; using a kit from Roch (Germany), measured automatically with Minividas, Biomerieux (France). The results were computed as the mean \pm standard error. In all statistical analyses, only $\mathrm{p} \leq$ 0.05 was considered significant. Differences among groups were analyzed by variance oneway analysis ANOVA followed by Fisher's test for multiple comparisons, using Statview version 5.0. Differences were considered significant when $\mathrm{p}<0.05$. Regression analysis was performed by analysis of covariance (ANCOVA) also using Stat view version 5.0.

\section{Results and Discussion}

There are no differences between levels of myocardial markers in RA patients comparing with the healthy group included troponin, creatinine kinase, GOT, and Lactate dehydrogenase. Moreover, there are no differences between these enzyme levels in male and female groups. However, they were within normal values which are as following: (troponin $=0-0.4 \mathrm{ng} / \mathrm{ml}$, $\mathrm{CK}=22-198 \mathrm{U} / \mathrm{L}$, GOT=8-45U/L, and LDH=140-280U/L) (Figure 1). 


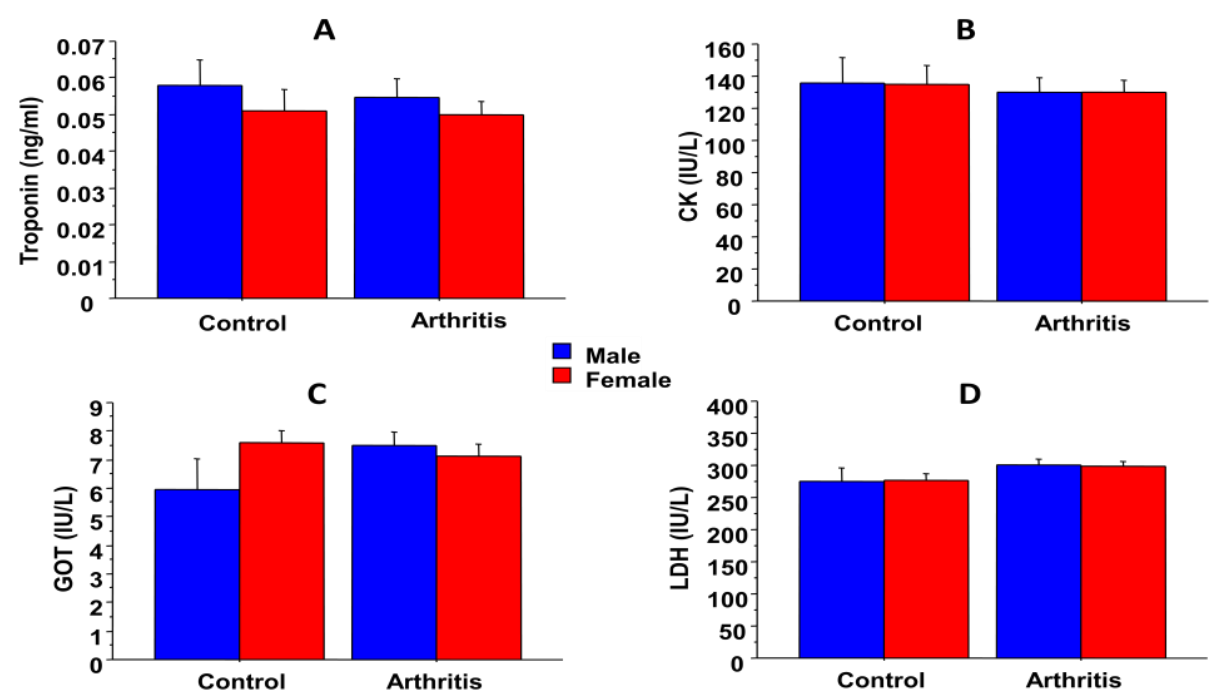

Figure 1. Myocardial markers levels in rheumatoid arthritis patients and control groups. (A) troponin, (B) creatinine kinase (CK), (C) glutamic oxaloacetic transaminas GOT, (D) Lactate dehydrogenase (LDH).

Figure 2 shows lipid profile levels in both RA patients and healthy groups (male and female). There were no differences in all lipid profiles levels of control and RA patients groups however they were within normal values which are as following: (cholestrol $<150 \mathrm{mg} / \mathrm{dl}$, triglyceride $<150 \mathrm{mg} / \mathrm{dl}, \quad \mathrm{HDL}=40-59 \mathrm{mg} / \mathrm{dl}, \quad \mathrm{VLDL}=2-30 \mathrm{mg} / \mathrm{dl}$, and $\mathrm{LDL}<100 \mathrm{mg} / \mathrm{dl})$. Moreover, there are no differences between these enzyme levels in male and female groups. Interestingly, the cholesterol / HDL ratio was also calculated. It increased significantly from $(2.53 \pm 0.027)$ in control to $(3.78 \pm 0.26)$ in RA patients.

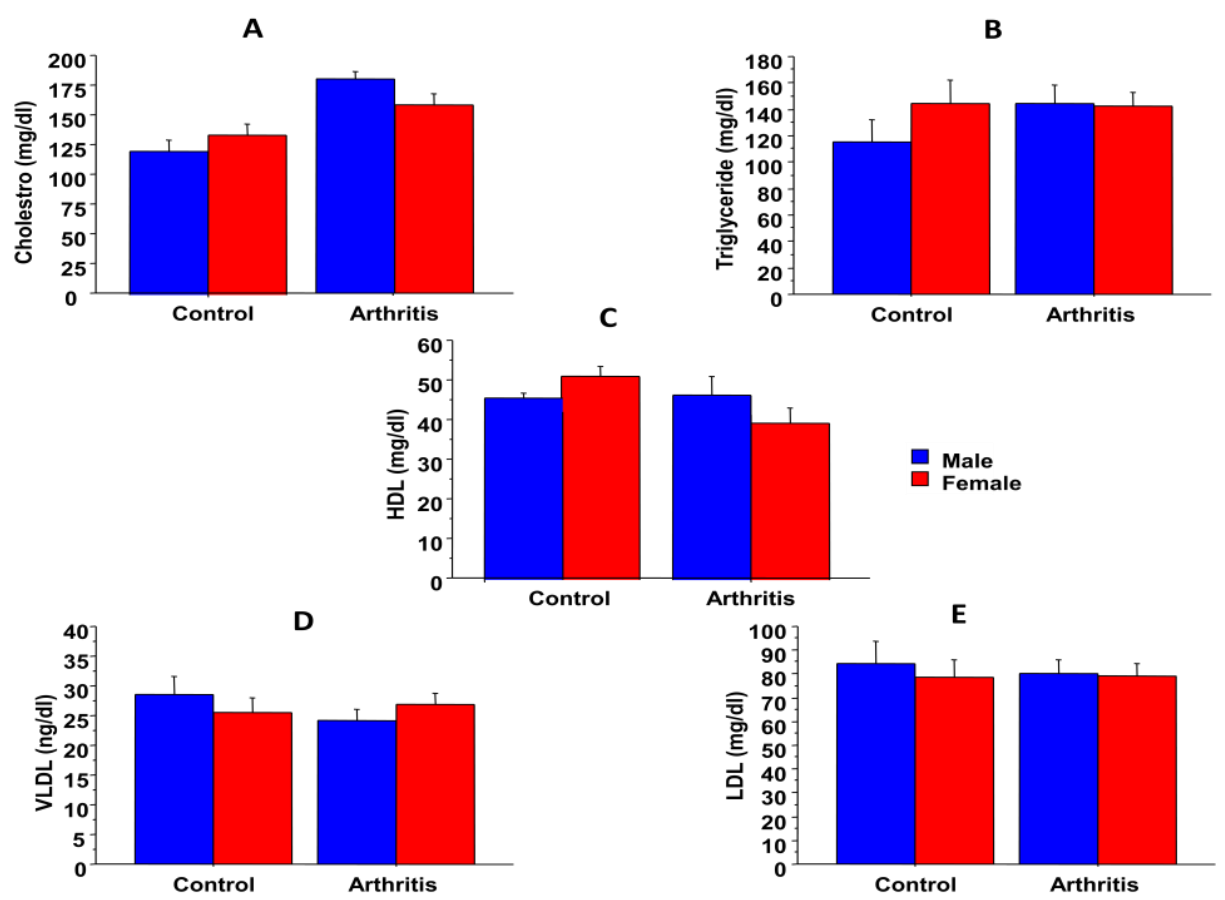

Figure 2. Lipid profiles levels in rheumatoid arthritis patients and control groups. (A) cholestrol, (B) triglyceridle, (C) high-density lipoprotein (HDL), (D) very-low-density lipoprotein (VLDL), (E) low-density lipoprotein (LDL).

The correlation between all these markers and age was also calculated. There were only significantly positive correlations between cholesterol and triglyceride with age whereas there For more information about the Conference please visit the websites: 
was no correlation between other markers and age (Table 1).

Table 1. the correlation between lipid profiles and myocardial markers.

\begin{tabular}{|c|c|c|c|}
\hline \multicolumn{2}{|c|}{ Parameters } & \multicolumn{2}{|c|}{ Age } \\
\cline { 2 - 4 } \multicolumn{2}{|c|}{} & R & 0.198 \\
\hline \multirow{3}{*}{ Myocardial Markers } & Troponin & 0.134 & 0.455 \\
\cline { 2 - 4 } & CK & 0.077 & 0.890 \\
\cline { 2 - 4 } & GOT & 0.01 & 0.247 \\
\cline { 2 - 4 } & LDH & 0.122 & 0.049 \\
\hline \multirow{3}{*}{ Lipid Profils } & Cholestrol & 0.219 & 0.002 \\
\cline { 2 - 4 } & Triglyceride & 0.305 & 0.946 \\
\cline { 2 - 4 } & HDL & 0.01 & 0.687 \\
\cline { 2 - 4 } & LDL & 0.055 & 0.687 \\
\cline { 2 - 4 } & VLDL & 0.045 & \\
\cline { 2 - 4 } & &
\end{tabular}

\section{Discussion}

Since RA patients have a higher rate of cardiovascular disease risk compared to individuals without autoimmune diseases [10], Periodic examination of cardiovascular risk according to the national guidelines used for the care of RA patients [11]. However, the general risk calculators used do not succeed in capturing the increased risks in these patients $[12,13]$.

Cardiovascular disease risk is associated with Many mechanisms, for example, the adverse effects of pharmacological RA, RA treatment, and accelerated atherosclerosis due to inflammatory processes associated with long-term RA [14-17]. In our work, all patients were newly diagnosed with rheumatoid arthritis, so this could be the reason why there wasn't a difference between cardiac parameters levels in RA patients compared with healthy groups. Kremers et al and Lindhardsen et al also stated that the cardiovascular disease risk in RA patients was similar to that of people without rheumatoid arthritis [7, 18]. But this result does not exclude the possibility of an increase in cardiovascular disease risk after 5-10 years of rheumatoid arthritis, although intensive treatment aimed at controlling inflammation in recent years is likely to decrease the cardiovascular disease risk rate that increases during the period of rheumatoid arthritis [19].

It is known that the lipid profile is one of the signs of cardiovascular disease because it makes rheumatoid arthritis patients triple cardiovascular disease risk. After all, this disease itself caused atherosclerosis (1). Lipid levels seem to change due to rheumatoid arthritis activity. Data of total cholesterol and low-density cholesterol concentrations differ in RA patients. Some works show similar levels [20] or less [21] of total cholesterol, however other researches indicated high concentration of total cholesterol and LDL cholesterol in early RA patients [22] or indicate decreased total cholesterol, LDL, and HDL inactive untreated RA patients $[6,21,23]$. In our results, there was no difference in all lipid profiles between RA patients and the health group.

Regardless of the changes in total cholesterol and HDL in RA patients, several studies support the calculating importance of total cholesterol to HDL ratio in RA patients, which leads to a more fatty arteriosclerosis profile and is associated with disease activity and

For more information about the Conference please visit the websites: 
improves after treatment with antirheumatic drugs $[20,21,22,24]$. In our results, the cholesterol/HDL ratio increased significantly in the RA patients than in the control group.

A similar gender-based pattern of cardiovascular disease risk observed in this study was reported by [25] and [7], who also found no general difference in cardiovascular disease risk between genders whereas there weren't differences in lipid profile and myocardial markers between women and men with RA in this study.

\section{Conclusion}

In conclusion, the cardiovascular disease risk could be increase also in the patient with newly diagnostic rheumatoid arthritis so other patient characteristics, such as gender, age at the onset of the disease, disease severity markers, and TC / HDL ratio should be probably included in a model to predict the cardiovascular disease risk for RA, developing such a model, validating it and assessing its primary and secondary prevention strategies for cardiovascular disease in rheumatoid arthritis patients.

\section{References}

1. Erum, U.; Ahsan, T; Khowaja, D. Lipid abnormalities in patients with Rheumatoid Arthritis. Pak. J. Med. Sci. 2017, 33, 1, 227-230.

2. Avina-Zubieta, J.A.; Thomas, J.; Sadatsafavi, M.; Lehman, A.J.; Lacaille, D. Risk of incident cardiovascular events in patients with rheumatoid arthritis: a meta-analysis of observational studies. Ann. Rheum. Dis. 2012 ,71, 9, 1524-1529.

3. Pant, S.; Deshmukh, A.; Neupane, P.; Kumar, M.P.K.; Vijayashankar, C.S. cardiac Biomarkers. In: Lakshmandoss U, editor. Noval strategies in ischemic heart disease. India: Mercy Health 2012, 17-42.

4. Gabriel, S.E.; Crowson, C.S. Risk factors for cardiovascular disease in rheumatoid arthritis. Curr. Opin. Rheumatol. 2012 ,24, 2, 171-176.

5. Boyer, J.F.; Gourraud, P.A.; Cantagrel, A.; Davignon, J.L.; Constantin, A. Traditional cardiovascular risk factors in rheumatoid arthritis: a meta-analysis. Joint Bone Spine. 2011 78, 2, 179-183.

6. Choy, E.; Sattar, N. Interpreting lipid levels in the context of high-grade inflammatory states with a focus on rheumatoid arthritis: a challenge to conventional cardiovascular risk actions. Ann. Rheum. Dis. 2009, 68, 4, 460-469.

7. Lindhardsen, J.; Ahlehoff, O.; Gislason, G.H.; Madsen, O.R.; Olesen, J.B.; TorpPedersen. C. The risk of myocardial infarction in rheumatoid arthritis and diabetes mellitus: a Danish nationwide cohort study. Ann. Rheum. Dis. 2011 ,70, 6, 929-934.

8. Steiner, G.; Urowitz. M.B. Lipid profiles in patients with rheumatoid arthritis: mechanisms and the impact of treatment. Semin Arthritis Rheum. 2009 ,38, 5, 372-381.

9. Amezaga-Urruela, M.; Suarez-Almazor, M.E. Lipid paradox in rheumatoid arthritis: changes with rheumatoid arthritis therapies. Curr Rheumatol Rep. 2012 , 14, 5, 428-437.

10. Naranjo, A.; Sokka, T.; Descalzo, M.A.; Calvo-Alen, J.; Horslev-Petersen, K.; Luukkainen. R.K. Cardiovascular disease in patients with rheumatoid arthritis: results from the QUEST-RA study. Arthritis Res. Ther. 2008 , 10, 2, R30.

11. Agca, R.; Heslinga, S.C.; Rollefstad, S.; Heslinga, M.; McInnes, I.B.; Peters, M.J. EULAR recommendations for cardiovascular disease risk management in patients with rheumatoid arthritis and other forms of inflammatory joint disorders: 2015/2016 update. Ann Rheum Dis. 2017 , 76, 1, 17-28.

For more information about the Conference please visit the websites: 
12. Crowson, C.S.; Matteson, E.L.; Roger, V.L.; Therneau, T.M.; Gabriel, SE. Usefulness of risk scores to estimate the risk of cardiovascular disease in patients with rheumatoid arthritis. Am J Cardiol. 2012 , 110, 3, 420-424.

13. Siontis, G.C.; Tzoulaki, I.; Siontis, K.C.; Ioannidis, J.P. Comparisons of established risk prediction models for cardiovascular disease: systematic review. Bmj. 2012 ,344, e3318.

14. Gonzalez, A.; Maradit-Kremers. H.; Crowson. C.S.; Ballman. K.V.; Roger. V.L.; Jacobsen, S.J. Do cardiovascular risk factors confer the same risk for cardiovascular outcomes in rheumatoid arthritis patients as in non-rheumatoid arthritis patients? Ann Rheum Dis. 2008 ,67, 1, 64-69.

15. Solomon, D.H.; Curhan, G.C.; Rimm, E.B.; Cannuscio, C.C.; Karlson, E.W. Cardiovascular risk factors in women with and without rheumatoid arthritis. Arthritis Rheum. 2004 ,50, 11, 3444-3449.

16. Toms, T.E.; Symmons, D.P.; Kitas, G.D. Dyslipidaemia in rheumatoid arthritis: the role of inflammation, drugs, lifestyle and genetic factors. Curr Vasc Pharmacol. 2010, 8, 3, 301-326.

17. Solomon, D.H.; Kremer, J.; Curtis, J.R.; Hochberg, M.C.; Reed, G.; Tsao, P. Explaining the cardiovascular risk associated with rheumatoid arthritis: traditional risk factors versus markers of rheumatoid arthritis severity. Ann Rheum Dis. 2010 ,69, 11, 1920-1925.

18. Kremers, H.M.; Crowson, C.S.; Therneau, T.M.; Roger, V.L.; Gabriel, S.E. High ten-year risk of cardiovascular disease in newly diagnosed rheumatoid arthritis patients: a population-based cohort study. Arthritis Rheum. 2008 ,58, 8, 2268-2274.

19. Avina-Zubieta, J.A.; Choi, H.K.; Sadatsafavi, M.; Etminan, M.; Esdaile, J.M.; Lacaille, D. Risk of cardiovascular mortality in patients with rheumatoid arthritis: a meta-analysis of observational studies. Arthritis Rheum. 2008 59, 12, 1690-1697.

20. Park, Y.B.; Lee, S.K.; Lee, W.K.; Suh, C.H.; Lee, C.W.; Lee, C.H,. Lipid profiles in untreated patients with rheumatoid arthritis. J. Rheumatol. 1999 ,26, 8, 1701-1704.

21. Boers, M.; Nurmohamed, M.T.; Doelman, C.J.; Lard, L.R.; Verhoeven, A.C.; Voskuyl. A.E. Influence of glucocorticoids and disease activity on total and high density lipoprotein cholesterol in patients with rheumatoid arthritis. Ann Rheum Dis. 2003, 62, 9, 842-845.

22. Georgiadis, A.N.; Papavasiliou, E.C.; Lourida, E.S.; Alamanos, Y.; Kostara, C.; Tselepis. A.D. Atherogenic lipid profile is a feature characteristic of patients with early rheumatoid arthritis: effect of early treatment--a prospective, controlled study. Arthritis Res. Ther. $2006,8,3, \mathrm{R} 82$.

23. Myasoedova, E.; Crowson, C.S.; Kremers, H.M.; Roger, V.L.; Fitz-Gibbon. P.D.; Therneau. T.M. Lipid paradox in rheumatoid arthritis: the impact of serum lipid measures and systemic inflammation on the risk of cardiovascular disease. Ann Rheum Dis. 2011 ,70, 3, 482-487.

24. van Halm, V.P.; Nielen, M.M.; Nurmohamed, M.T.; van Schaardenburg, D.; Reesink. H.W.; Voskuyl, A.E. Lipids and inflammation: serial measurements of the lipid profile of blood donors who later developed rheumatoid arthritis. Ann Rheum Dis. 2007, 66, 2, 184188.

25. Solomon, D.H.; Goodson, N.J.; Katz, J.N.; Weinblatt, M.E.; Avorn, J.; Setoguchi, S. Patterns of cardiovascular risk in rheumatoid arthritis. Ann Rheum Dis. 2006,65, 12, 16081612. 